\title{
A facile route for controlled alignment of carbon nanotube-reinforced, electrospun nanofibers using slotted collector plates
}

\author{
G. R. Rakesh, G. S. Ranjit, K. K. Karthikeyan, P. Radhakrishnan, P. Biji* \\ Nanosensor Laboratory, PSG Institute of Advanced Studies, Coimbatore-641004, India
}

Received 12 April 2014; accepted in revised form 10 September 2014

\begin{abstract}
A facile route for controlled alignment of electrospun multiwalled carbon nanotube (MWCNT)-reinforced Polyvinyl Alcohol (PVA) nanofibers using slotted collector geometries has been realized. The process is based on analytical predictions using electrostatic field analysis for envisaging the extent of alignment of the electrospun fibers on varied collector geometries. Both the experimental and theoretical studies clearly indicate that the introduction of an insulating region into a conductive collector significantly influences the electrostatic forces acting on a charged fiber. Among various collector geometries, rectangular slotted collectors with circular ends showed good fiber alignment over a large collecting area. The electrospun fibers produced by this process were characterized by Atomic Force Microscopy (AFM), High Resolution Transmission Electron Microscopy (HRTEM), Scanning Electron Microscopy (SEM) and Optical Microscopy. Effects of electrospinning time and slot widths on the fiber alignment have been analyzed. PVA-MWCNT nanofibers were found to be conducting in nature owing to the presence of reinforced MWCNTs in PVA matrix. The method can enable the direct integration of aligned nanofibers with controllable configurations, and significantly simplify the production of nanofibersbased devices.
\end{abstract}

Keywords: nanocomposites, electrospinning, electrostatic field analysis, carbon nanotubes, aligned nanofibers

\section{Introduction}

Nanowires with tailored physical properties have been used as key components in flexible electronics, electronic logic gates, renewable energy technologies, and chemical/biological sensor applications [1]. Direct integration of nanowires in devices remains as a major challenge, as it is difficult to reproducibly locate them in specific positions and orientations [2]. Development of cost-effective and reliable techniques for controlled integration of nanowires onto devices is very much essential for the commercialization of nanotechnology related products [3, 4]. Among various nanofabrication techniques, electrospinning is a highly versatile and cost-effective process in which, solid micro and nanofibers are produced from a polymeric fluid stream (solution or melt) delivered through a nozzle $[5,6]$. Several innovative approaches have been introduced in this field for developing nanofibers with varied constitution and structure for diverse applications. There have been much research and progress in the development of various designs and modification to the electrospinning process over the last century. Special multi-spinneret designs, such as, coaxial, bicomponent, gas-jacketed electrospinning have made a breakthrough in the electrospinning process by creating hybrid electrospun nanofibers [7-11].

One of the major problems that influence the commercial viability of nanofibers membranes is the

\footnotetext{
${ }^{*}$ Corresponding author, e-mail: bijuja123@yahoo.co.in

(C) BME-PT
} 
lack of structural integrity of ultrathin electrospun membranes. The full potential of electrospun nanofibers can be utilized for commercial applications, only when they are assembled as well-ordered structures with the desired mechanical stability and structural integrity. Hierarchically organized nanofibers have great potential to solve this problem, as they allow interfacing nanofibers with various structural surfaces $[12,13]$. So far, the challenge of controlling the spatial orientation of electrospun fibers has been met with partial success [7, 14]. Both mechanical and electrostatic means have been explored to improve the alignment of electrospun nanofibers [15-17]. Many reports show that the asspun fibers could be aligned more or less parallel to each other when a rotating drum at high speed is used as the collector [18]. A wheel-like bobbin was used as the collector to position and align individual polymer nanofibers and was demonstrated by Zussman and co-workers. But, since the edge of such a bobbin had to be relatively sharp, this technique was not feasible for the formation of well-aligned nanofibers over large areas [19]. This problem has been resolved partially by parallel plate collectors which consist of two pieces of electrically conductive substrates separated by a gap to produce uniaxially aligned arrays of fibers [20]. This method was further extended by a simple method by Katta et al. [21] for spinning sheets with $1 \mathrm{~cm}$ wide strips of aligned nanofibers using copper wires spaced evenly in the form of a circular drum as a collector of the electrospun nanofibers. Another approach was developed by Chvojka et al. [22] who produced PVA nanoyarns using a special saw-like collector, by twisting electrospun PVA nanofibers and introduced a simple analysis of the field strength that causes the prevailing unidirectional fiber deposition between neighbouring lamellae of a special saw-like collector. Few groups have also worked on producing aligned fibers by using collector plates of special designs [23-25]. But, it is difficult to understand how the electric field behaves in each of these types of collector designs. Although numerous reports exist about the preparation and application of electrospun polymer nanofibers and composites, very small attention have been paid to how to manipulate the electric field for producing high quality or aligned nanofibers.

Carbon nanotubes (CNTs) have high Young's modulus, low density, and excellent electrical and ther- mal properties, which make them ideal fillers for polymer nanocomposites [26, 27]. Production of carbon nanotube-polymer nanocomposite materials is one of the key factors controlling the mechanical properties in composite and hybrid systems. Carbon nanotube-polymer nanocomposites in the form of nano/microfibers have immense applications, such as, nanofilters of scalable pore size and structural components for microdevices. Electrospun CNT/ polymer nanofibers have been demonstrated to be attractive for a large variety of potential applications, such as, in optoelectronic and sensor devices. Other potential applications of electrospun CNTs/polymer nanofibers include tissue engineering scaffolds, composite reinforcement, drug carriers for controlled release and energy storage [28, 29]. To realize their commercial applications, considerable work is still required. This includes a thorough understanding of the structure-property relationship for various electrospun polymer nanofibers, the effective incorporation of carbon nanotubes into polymer fibers with a high loading content, and large scale production of composite nanofibers of consistent and high quality, but at a low cost. Few reports prove that electrospinning a polymer solution containing well-dispersed carbon nanotubes lead to nanocomposite fibers. During fast fiber-drawing process, these fibers embed carbon nanotubes, oriented parallel to the nanofiber axis due to large shear forces [30,31]. A modified rotating disk collector including two separate parallel aluminium plates with sharp edges was used by Huang et al. [32] to fabricate SWNTs/ polyvinylidene difluoride (PVDF) composite nanofibers. They observed that the interfacial interaction between SWNTs and PVDF and the application of extensional forces have a strong synergistic effect on crystalline structures of PVDF inducing oriented crystallites at only $0.01 \mathrm{wt} \%$ of nanotubes. Ge and coworkers developed oriented composite nanofiber sheets made from surface-oxidized multiwalled carbon nanotubes (MWCNTs) and polyacrylonitrile (PAN) using electrospinning [29]. Recently, Chen et al. [33] demonstrated that surface-functionalized MWNTs were homogeneously dispersed and highly aligned along the polyimide nanofiber axis, whereas most of the pristine MWNTs form aggregates or bundles and even protruded out of the electrospun nanofibers. Zhang et al. [34] produced uniaxially aligned electrospun nanofibers of PAN and Triton X-100 grafted MWNTs using a collector consisting 
of two pieces of electrodes as well as a slowly rotating drum with multi-electrodes. Yee et al. [35] modified the design of a disk by using two separate parallel aluminium electrodes attached to a rotating disk to collect the well-aligned MWCNT/PVDF nanofibers, where both the electric field and mechanical force contributed to nanofiber alignment.

Though there are various special dynamic collectors reported, such as, rotating drums, wire drums, discs, rings etc., most of them require an external power to rotate at high rpm and it is difficult to deposit the aligned fibers onto devices directly [7]. Static collectors includes parallel strips, micromachined patterns etc., where aligned fiber formation is possible, but, mechanical stability for the aligned fibers is very poor, especially while integrating these fibers onto devices [36, 37]. Hence, there is a need for developing low cost, efficient techniques to fabricate aligned nanofibers. In this investigation, we report a simple and novel route to predict the fiber alignment to produce uniaxially aligned MWCNT-reinforced PVA nanofibers on various collector geometries. The fundamental concept adopted for this investigation is based on engineering the electrostatic field using slotted collectors during electrospinning. Our main emphasis here is to alter the nature of electrostatic field on collector plate by creating a repulsive field of various magnitude and directions by using collector plates having varied geometrical slots to produce aligned electrospun nanofibers. The collector geometries used in this investigation are having in-built insulating air gaps which induce fiber alignment by varying the electrostatic field lines. This method can provide proper mechanical stability to the fibers formed compared to the parallel plate collectors. It is also possible to use multiple slots made on single collector to produce aligned nanofibers. This method can be further used for direct integration of electrospun nanofibers having any composition to microdevices, especially for sensor applications. Current process can also be further extended in combination with near field electrospinning techniques for patterning various designs of nanofibers which is having immense applications in micro/nanoelectronics.

\section{Experimental details}

\subsection{Materials and methods}

Polyvinyl alcohol, (PVA, 99\%, $M_{\mathrm{W}}=140000$, HiMedia) was used without further purification to prepare feed solution for the electrospinning. Commercially available multiwalled carbon nanotubes (MWCNT, $>90 \%$, diameter: $20-30 \mathrm{~nm}$, length: $0.5-$ $2 \mu \mathrm{m}$, Quantum Materials Corp., India) produced by Chemical Vapour Deposition were used for the composite nanofiber formation. Ultrapure water (Millipore Academic, Resistivity, $18.2 \mathrm{M} \Omega \cdot \mathrm{cm}$ ) was used for the preparation of feed solution. Optical Microscopic images were obtained using Polarized Optical Microscope (Leica, UK). Atomic Force Microscopic (AFM) images were acquired using Multimode Scanning Probe Microscope (NTMDTNTEGRA, Russia) for the electrospun fibers transferred onto $p$-type $\mathrm{Si}(100)$ substrates. Transmission Electron Microscopic (TEM, JEOL 2010, Japan) analysis was carried out for the electrospun fibers transferred onto carbon coated copper films. Field Emission Scanning Electron Microscope (ZEISS SUPRA 55, Germany) was used to study the alignment and morphology of the nanofibers. Conductivity studies were carried out using a high-current source meter (Model: 2420, Keithley, USA).

Electrostatic field analysis was performed using COMSOL Multiphysics software. The CAD model for the collector plates were created in SOLIDWORKS software and imported to COMSOL Multiphysics. Aluminium plates (thickness: $3 \mathrm{~mm}$ ) were used to fabricate collector geometries by introducing slots of required geometry and dimensions. Different geometric configurations were created using wire electric discharge machining (EDM). EDM drilling was done prior to wire EDM process to provide starting holes for the wires. After machining, the plates were cut into required dimensions using shear cutter and cleaned ultrasonically using ethanol and ultrapure water. The cleaned, slotted collector plates were used for the electrospinning process without further surface treatments.

\subsection{PVA-MWCNT feed solution preparation}

The carbon nanotubes were functionalized using acid treatment as per previous reports to enhance the dispersion of carbon nanotubes in the polymer fibers [38]. Carboxylic and hydroxyl groups were produced at the peripheral walls of carbon nanotubes by this process and thereby achieved a good dispersion producing unbundled CNTs. Concentration of PVA in ultrapure water was kept constant as $10 \mathrm{wt} \%$ throughout the experiments after optimizing parameters, such as, voltage, needle-to-collector 
distance, flow rate and concentration. PVA-MWCNT feed solution was prepared by mixing $1 \mathrm{wt} \%$ MWCNT with $10 \mathrm{wt} \%$ PVA solution for electrospinning. Before electrospinning, the mixture was stirred and ultrasonicated (power: 500 watts, frequency: $20 \mathrm{kHz}$ ) for 30 minutes to avoid granules present in the solution and to obtain uniform dispersion of CNTs.

\subsection{Nanofiber production using electrospinning process}

The electrospinning of PVA-MWCNT was carried out using an in-house electrospinning unit fabricated in our laboratory. The experimental set-up was enclosed in Pyrex glass chamber to ensure operator safety while the process is in progress and to control air turbulence. A screw rod operated beam made of an insulating material was inserted inside the chamber so that height of the beam could be adjusted. The other column of the beam was firmly grounded on the table. A nozzle (20 gauge diameter) was inserted inside the chamber over the end of the beam in the provision provided using the screw type arrangement. The nozzle was made of stainless steel for electrical conductivity and mechanical strength. A Syringe pump (Larsen \& Toubro Ltd., Model No: SP102 with flow rate: $0.1-999 \mathrm{~mL} / \mathrm{hr}$ ) was used to feed the solution to the nozzle. A syringe $(20 \mathrm{~mL})$ was used as a piston and cylinder and is connected to the feed pump. A flexible tube $(3 \mathrm{~mm}$ diameter) was connected between the syringe and the nozzle. A regulated High Voltage DC power supply (Glassman High Voltage Inc., New Jersey, USA, Model No: EH30P3) with output voltage range $1-30 \mathrm{kV}$ was used for electrospinning. The positive terminal of the transformer was connected to the nozzle so that when the material is flowing it gets positively charged. The negative terminal of the transformer was connected to the collector plate. The electrospinning takes place in the gap between the nozzle and collector plate. The method was optimized by analyzing various combinations of electrospinning parameters, such as, polymer concentration, applied voltage, nozzle-collector distance, needle gauge and flow rate in order to obtain uniform nanofibers. All experiments were carried out under ambient conditions (temperature: $29^{\circ} \mathrm{C}, R H=$ $60 \%$ ). Optimized voltage used for this study was $25 \mathrm{kV}$ for $10 \mathrm{wt} \%$ PVA concentration mixed with $1 \mathrm{wt} \%$ MWCNTs with needle-to-collector distance of $20 \mathrm{~cm}$. Flow rate used was $0.8 \mathrm{~mL} / \mathrm{hr}$ using a 20 gauge needle.

\section{Results and discussion \\ 3.1. Electrostatic field analysis for the prediction of fiber alignment}

According to the literature, the electric field distribution plays a major role in controlling fiber alignment. In this scenario, a detailed study of electric field distribution for collector configuration is essential for understanding the phenomenon of fiber alignment. To probe this phenomenon the electrostatic field distributions of various collector geometries were plotted using electrostatic module of COMSOL Multiphysics software. A potential of $25 \mathrm{kV}$ was applied at the needle tip and the collector plate was grounded. The collector material and the medium surrounding the collector were modelled as aluminium and as air respectively. After subsequent meshing, the computation results were acquired. From the electrostatic field analysis, it was observed that creating a slot in a collector plate introduced an insulating air gap which significantly alters the electric field. To study the influence of such insulating air gaps on electrostatic field distribution, the collector plates were modelled with slots having varied geometries (triangle, square, rectangle, semicircle and circle). The corresponding electrostatic fields are depicted in Figure 1. By analyzing the electric field plots, the region of fiber alignment within the slot geometry could be predicted. The analytical predictions were compared with electrospun fibers obtained experimentally on collector plates to estimate the accuracy of predictions. Figure 1a shows the electrostatic field pattern in a square slot. The field arrows are parallel at the middle of the square. The field near the corners could be resolved in order to obtain parallel field along lines perpendicular to the bisector of the vertex. Hence, the fibers are expected to be aligned at the middle of the slot and at the corners. The fibers follow the direction of the lines perpendicular to the bisector of the vertex. As depicted in Figure 1b, the field distribution within the collector with a rectangular slot is very much similar to that of the parallel plate electrodes reported in literature. Hence better alignment of the fibers is expected in the middle of the slot, except at the extreme edges where the field is randomly oriented. Figure 1c represents the electric field lines in a rectangular slot with circular ends. In this case, the lateral com- 


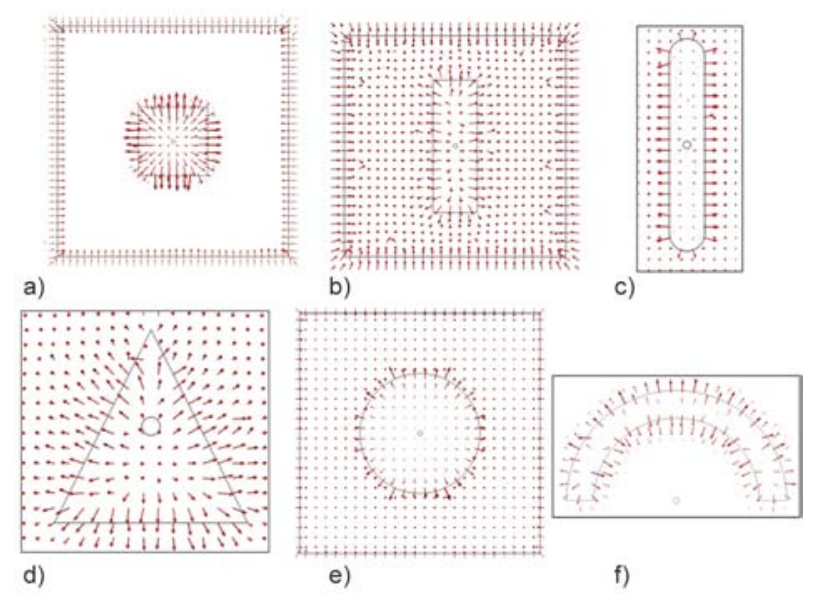

Figure 1. Electric field distribution in various geometric slots: (a) square, (b) rectangular, (c) rectangular slots with circular ends, (d) triangular (e) circular and (f) semi annular slots

ponent is radial near the circular ends and parallel in the rectangular portion. The parallel directions pointing outward indicate repulsive field in the slotted portion. This parallel repulsive electrostatic field is similar to that of the parallel plate collectors reported in literature and hence good fiber alignment is expected in the rectangular region. But, at the ends there is no such uniform field exists and hence random fiber orientation is expected. The electric field plot for triangular slot in Figure 1d indicates that the field tends to bend at the edges creating a lateral component. The combined effect of the lateral component of the two intersecting edges induces the fiber alignment. The electrostatic field at the corners could be resolved to obtain a parallel repulsive field in the direction normal to the bisector of the vertex. Thus, the fibers are expected to be aligned in the direction normal to the bisector of the vertex. Since the field direction is axial at the center of the triangle fiber formation in this region is difficult. The electrostatic field in circular slot shown in Figure 1e is uniformly radial in all directions; therefore the fibers are expected to be straight without any curvature but could not be aligned in a particular direction since the field is uniform in all directions. Figure $1 \mathrm{f}$ depicts electrostatic field in the semi annular slot indicating radial electric field distribution. The lateral component of the field is parallel between the slots and hence, reasonable fiber alignment is expected in the slotted portion. The fiber alignment direction is supposed to be radial in accordance with the electric field. According to the electrostatic field analysis, among all the geometrical slots used for this study, rectangular slots with circular end showed maximum alignment of nanofibers in larger area compared to other geometries, which was further confirmed from electrospinning experiments using various geometries. Hence, collectors having rectangular slot with circular ends was chosen for further studies. Figure 2 shows various geometrical collectors (semi annular, triangular, square, rectangular and spherical) used for this study and the corresponding optical microscopic images of the electrospun PVA-MWCNT fibers which was found to be in accordance with electrostatic field analysis.

From the electrostatic analysis, it is evident that the electric field lines tend to bend near the slot edges

Fabricated collector geometries

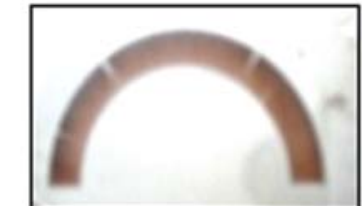

a)

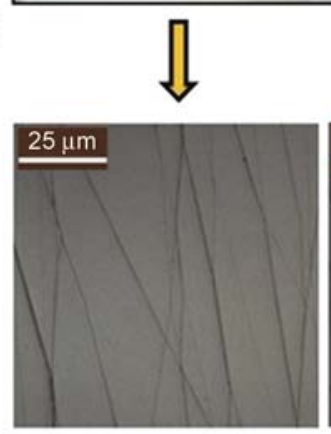

b)
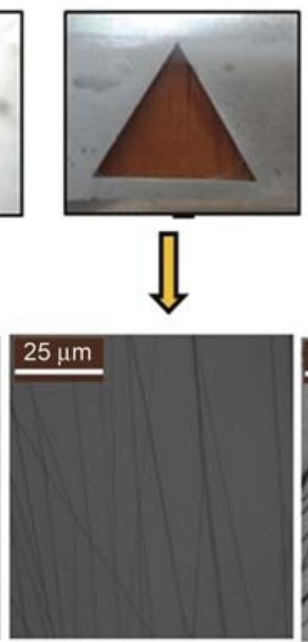

Optical images of electrospun PVA-CNT composite fibers

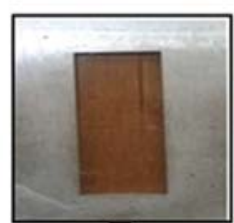

$\prod$
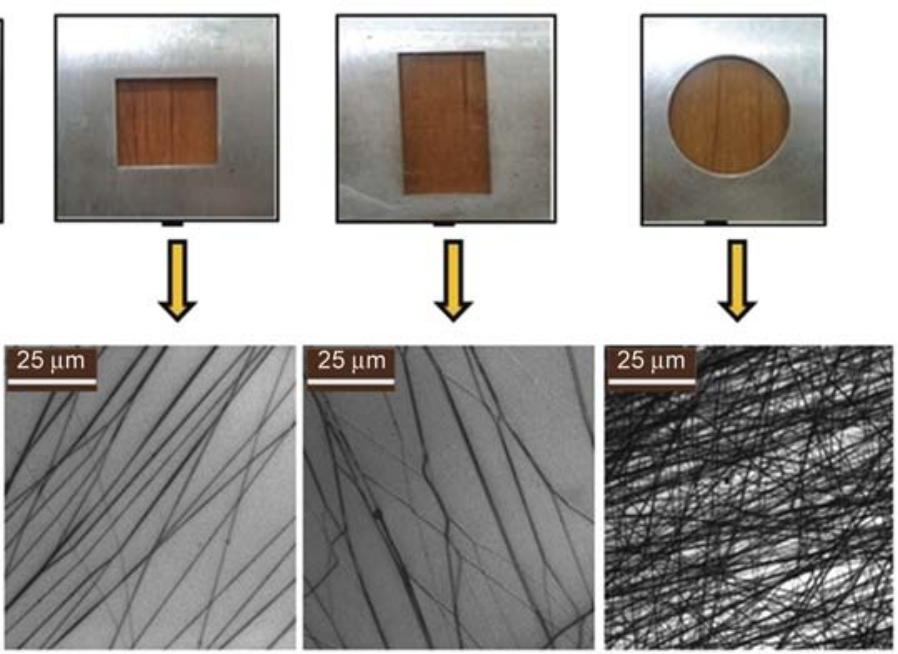

Figure 2. (a) Photographs of various geometrical collectors (semi annular, triangular, square, rectangular and spherical) and (b) corresponding optical microscopic images of the electrospun PVA-MWCNT fibers 
in rectangular slot with circular edges as depicted in Figure 3b, 3c (the arrows near the slot edges were slightly bent, whereas arrows indicate the direction of the electric field). This bending of field lines creates a lateral component of the electric field in the plane of collector plate. The direction of the lateral component depends upon the geometry of the slot. From this analysis, the alignment direction of the nanofibers could be easily predicted. Hence, it is expected from this study that for a rectangular slot with circular ends, the parallel portions can produce aligned nanofibers, whereas, the circular ends cannot produce any aligned nanofibers. Further experiments using electrospinning proved that this prediction is valid and explained in the following sections. The as-produced electrospun nanofiber can be considered as a string of positively charged elements connected through a viscoelastic medium [39]. As
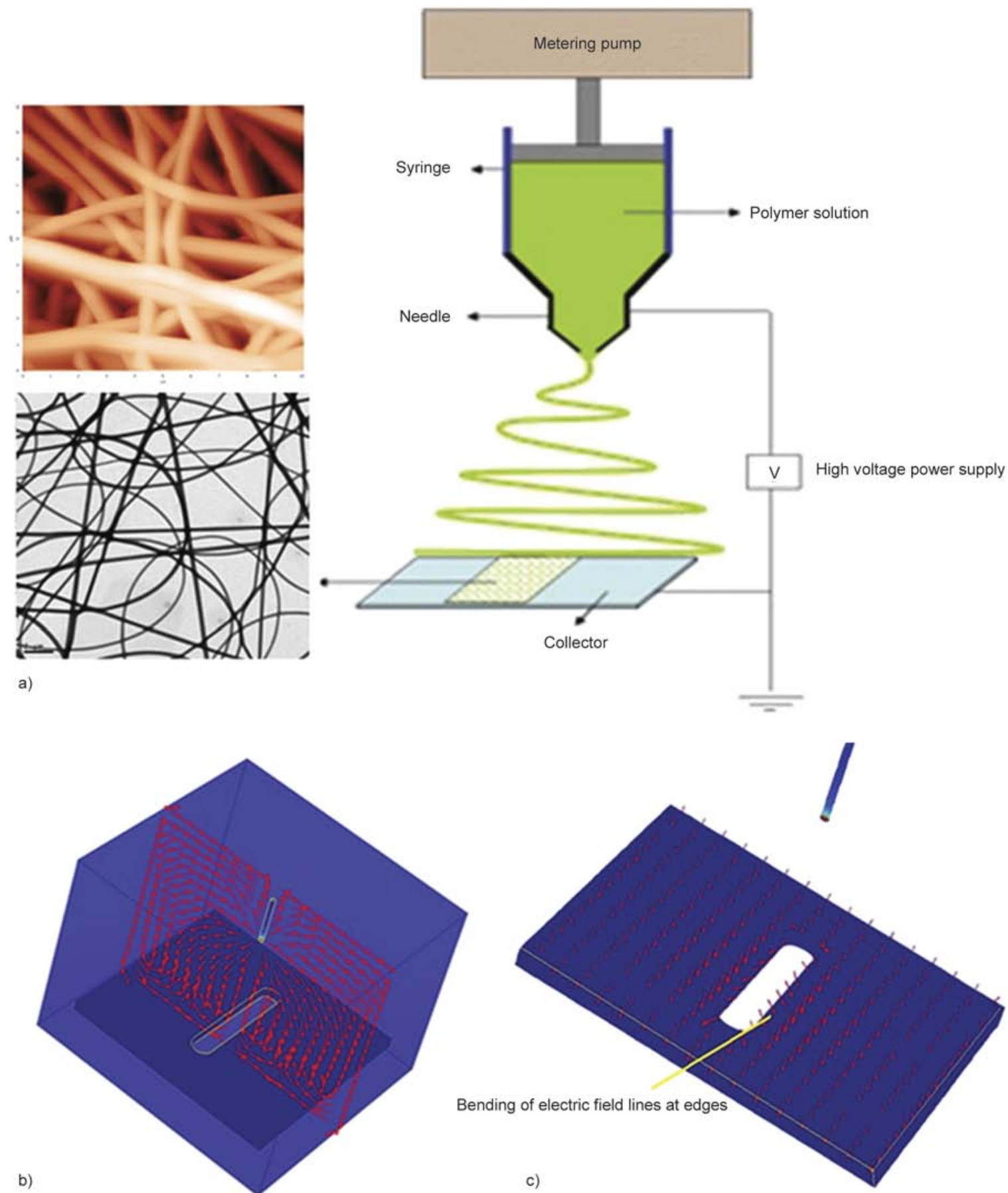

Figure 3. (a) Schematic representation of electrospinning (b) 3D view of electric field of rectangular slot with circular ends and (c) bending of electric field lines at edges of the slots 
the charged fibers approach the vicinity of the electrodes, charges on the fiber start inducing opposite charges on the surfaces of the electrodes. Since Coulomb interactions are inversely proportional to the square of the separation between charges, the two ends of the fiber closest to the electrodes should generate the strongest electrostatic force. This force stretches the nanofiber across the gap, perpendicular to the edges of the electrode. Though the fibers deposited directly on the electrode get immediate discharge, those present in the air slots remain charged. Thus, the electrostatic repulsion between the deposited and the upcoming fibers enhances the alignment process.

\subsection{Microscopic analysis of PVA-MWCNT composite nanofibers}

Figure $3 \mathrm{a}$ depicts the schematic representation of the electrospinning process used for the fabrication of nanofibers. Inset shows the representative AFM and TEM images of the PVA-MWCNT nanofibers formed on a flat aluminium collector plate without any geometrical slots. This clearly shows the random orientation of the PVA-MWCNT nanofibers on the collector plate. The feed solution used for electrospinning was an aqueous dispersion of $10 \mathrm{wt} \% \mathrm{PVA}$ and $1 \mathrm{wt} \%$ MWCNT. In order to confirm the presence of MWCNTs embedded in PVA nanofibers, the PVAMWCNT composite fibers were first electrospun onto carbon coated copper grids for high resolution transmission electron microscopic analysis. Figure 4 depict a comparative analysis of bare PVA nanofibers and PVA-MWCNT composite nanofibers produced directly on TEM grid by electrospinning using HRTEM. Figure $4 a, 4 b$ shows the TEM images of bare PVA nanofibers having diameter in the range of $180-380 \mathrm{~nm}$. The diameter of PVA-MWCNT was found to be decreased slightly $(100-345 \mathrm{~nm})$ with the loading of MWCNT as depicted in Figure 3c-3f. The high resolution images show the presence of embedded multiwalled carbon nanotubes in the electrospun PVA nanofibers as highlighted in Figure $4 \mathrm{~d}-4 \mathrm{f}$. The diameter of embedded carbon nanotubes was found to be $\sim 20 \mathrm{~nm}$. In order to verify the analytical predictions based on electrostatic field distribution discussed in the above section, electrospinning experiments were carried out using the collector plates having varied slot geometries machined using EDM. The as-produced fibers were electrospun onto each collector geometries and were characterized using TEM, SEM and Optical microscopy. Figure 5 depict the SEM images of the fibers electrospun on rectangular slotted collector with rounded ends. It is evident that the direction of fiber alignment is parallel between the two opposite edges of the slot. The fibers were found to be well-aligned at the centre region of the slot as indicated in Figure $5 \mathrm{a}-5 \mathrm{c}$ and at the rounded end portion significant alignment could not be achieved due to the intermixing of radial alignment (Figure $5 \mathrm{~d}$ ). The results were matching well with the electric field predictions performed on the rectangular slotted collectors with circular ends. The fiber alignment at the edge of the slot also was not satisfactory as it represents the zone of transition from random fiber orientation over the flat portion of the collector to the well aligned region between the slots. The radial distribution of the field at the rounded end portions did not cause any significant alignment of fibers. In addition to this, the residual charges of the previously formed fibers may also influence the fiber alignment. Effect of spinning time was studied to infer how the thickness of the mat influences the alignment of nanofibers. PVA-MWCNT nanofibers were collected after electrospinning for 1, 5, 10, 20 and 30 minutes. Figure 6a-6e shows the SEM images of PVA-MWCNT nanofibers collected using $10 \mathrm{~mm}$ rectangular slotted collectors with circular ends with increasing electrospinning time until 30 minutes. It is evident that with increase in the electrospinning time, the degree of alignment was decreasing. Figure 6 f shows the SEM image of bare PVA nanofibers collected after 10 minutes of electrospinning. Table 1 summarises the effect of electrospinning time on the degree of alignment of the nanofibers. The analysis of fiber orientation also shows that the degree of orientation of the nanofibers was not significantly affected by MWCNT loading, but it definitely decreased the diameter of the composite nanofibers formed as clear from Figure 5. The slight decrease in diameter for PVA-MWCNTs nanofibers compared to bare PVA nanofibers during alignment may be due to the contribution of the conductive components, MWCNTs reinforced in PVA. In addition, the hydrogen bonding interactions between the hydroxyl groups present in PVA and carboxyl groups present on the acid treated MWCNTs can also contribute to the reduction in PVA-MWCNT fiber diameter. The 

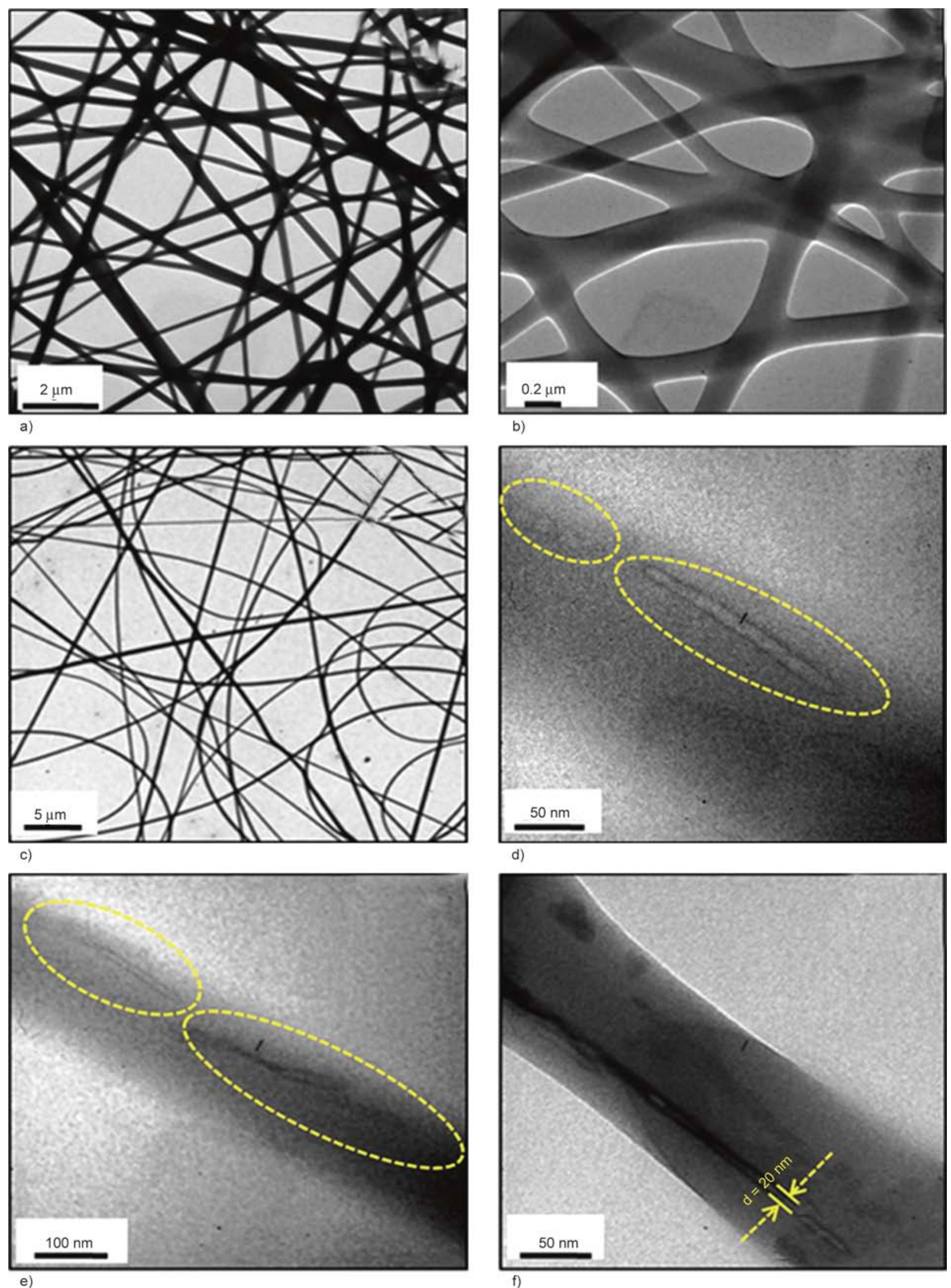

Figure 4. (a, b) Representative HR-TEM images of $10 \mathrm{wt} \%$ PVA nanofibers and (c-f) PVA-MWCNT (1 wt $\%)$ composite nanofibers produced by electrospinning process. Highlights: MWCNTs embedded in PVA nanofibers. 


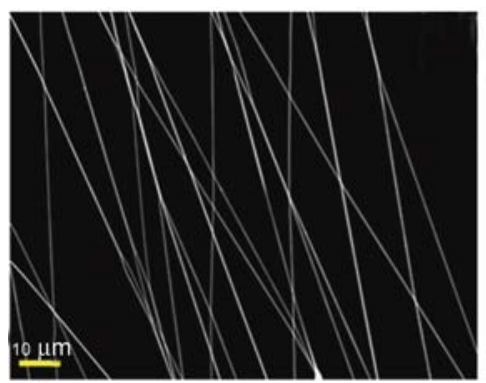

a)

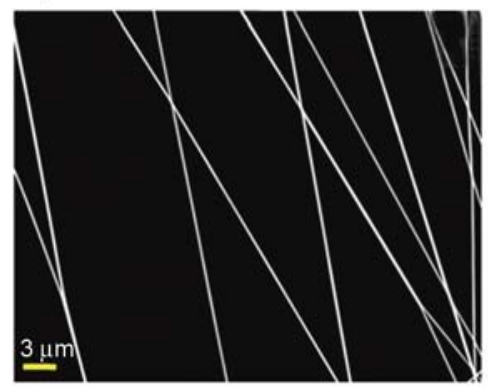

c)

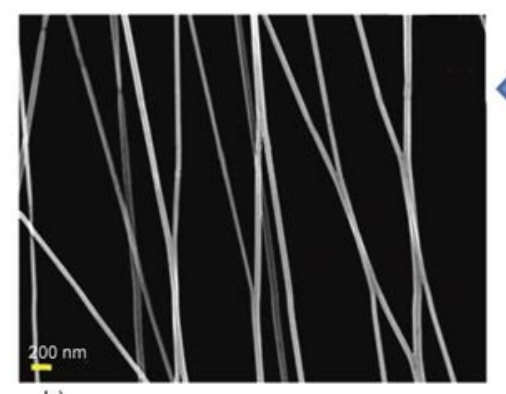

b)

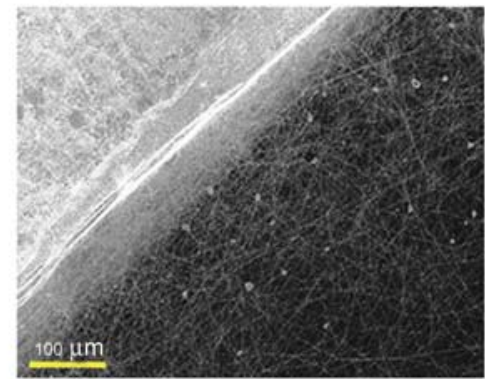

d)
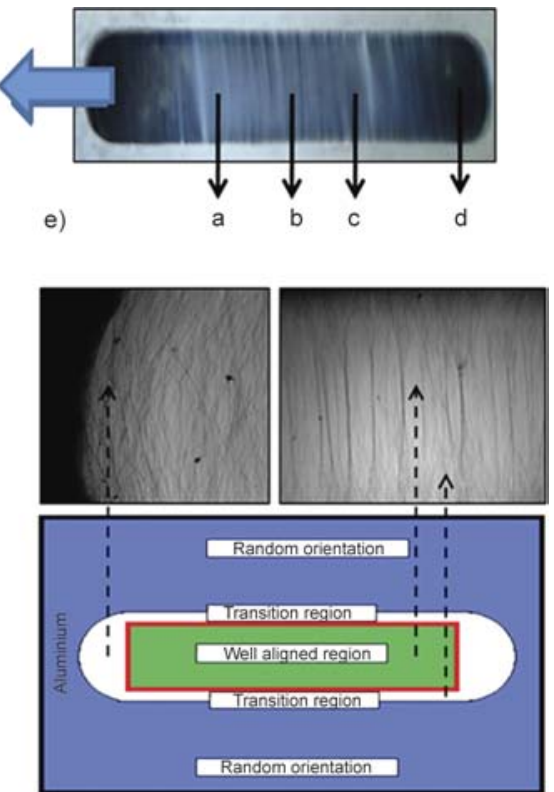

f)

Figure 5. Representative SEM images of $(a-d)$ the electrospun PVA-MWCNT composite nanofibers at varied places as indicated in (e) rectangular slot $(10 \mathrm{~mm})$ with circular ends and (f) schematic representation of regions of alignment on a rectangular slotted collector with circular ends and corresponding optical microscopic images

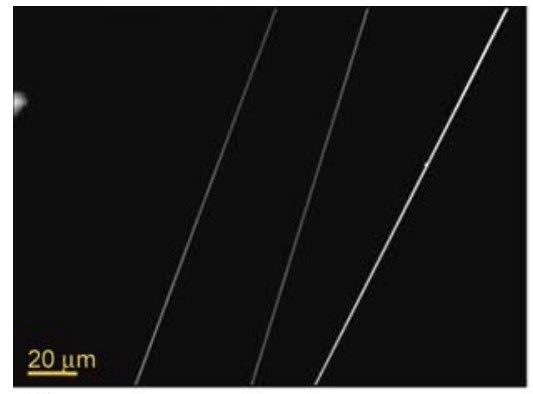

a)

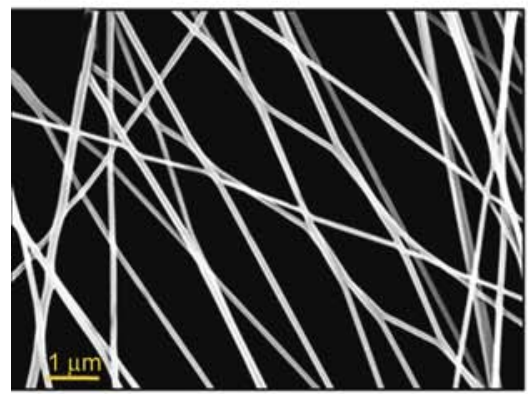

d)

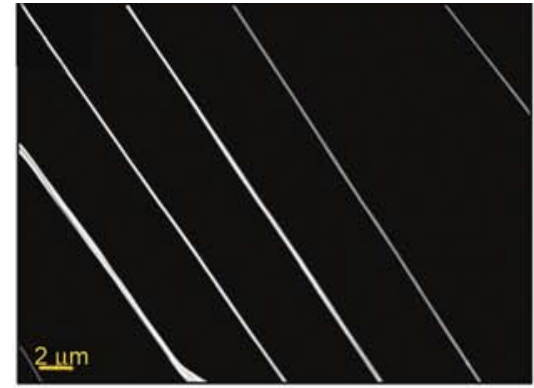

b)

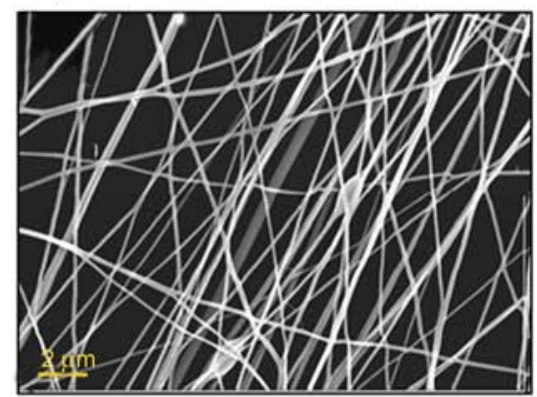

e)

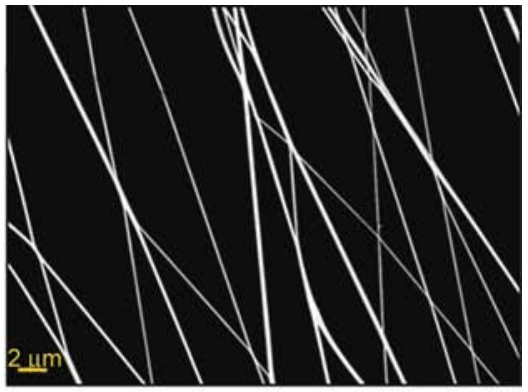

c)

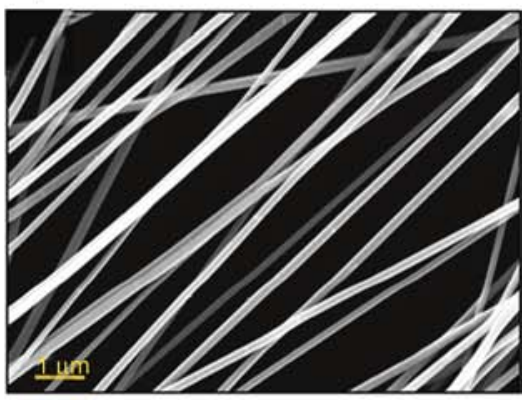

f)

Figure 6. SEM images of PVA-MWCNT nanofibers collected using $10 \mathrm{~mm}$ rectangular slotted collectors with circular ends for electrospinning time (a) $1 \mathrm{~min}$, (b) $5 \mathrm{~min}$, (c) $10 \mathrm{~min}$, (d) $20 \mathrm{~min}$, (e) $30 \mathrm{~min}$ and (f) bare PVA nanofibers collected for $10 \mathrm{~min}$

results indicate that the present technology can be used for aligned nanofiber integration onto devices, whereas, it is difficult to use this method for applications related to large area production of thick aligned mats.

Experiments were also performed by varying the slot widths of the rectangular slotted collectors with circular ends. It was observed that the fiber align- ment was good in slots of 5, 7.5 and $10 \mathrm{~mm}$, whereas, the fiber alignment was not satisfactory in the case of $20 \mathrm{~mm}$ slot. In the case of the collector with $20 \mathrm{~mm}$ slot, the slot width could be too long for the nanofibers to be supported between the slot edges. The fibers were unable to support in between the slot edges and were tending to break. The influence of the fiber diameter on slot width was studied after 
Table 1. Degree of alignment of PVA-MWCNTs and PVA nanofibers as a function of electrospinning time

\begin{tabular}{|c|c|}
\hline $\begin{array}{c}\text { Spinning time } \\
\text { [minutes] }\end{array}$ & $\begin{array}{c}\text { Degree of alignment } \\
{\left[{ }^{\circ}\right]}\end{array}$ \\
\hline 1 & 91 \\
\hline 5 & 88 \\
\hline 10 & 94 \\
\hline 20 & 89 \\
\hline 30 & 82 \\
\hline $10(\mathrm{PVA})$ & 90 \\
\hline
\end{tabular}

Table 2. Variation of average diameter as a function of slot width for rectangular slots with circular ends

\begin{tabular}{|c|c|c|}
\hline $\begin{array}{c}\text { Slot width } \\
{[\mathbf{m m}]}\end{array}$ & $\begin{array}{c}\text { Average fiber diameter } \\
{[\mathbf{n m}]}\end{array}$ & $\begin{array}{c}\text { Standard deviation } \\
{[\mathbf{n m}]}\end{array}$ \\
\hline 5 & 672 & 61.48 \\
\hline 7.5 & 399 & 74.37 \\
\hline 10 & 281 & 36.67 \\
\hline 20 & 265 & 42.48 \\
\hline
\end{tabular}

analyzing the corresponding SEM images. Figure 7 shows the representative SEM images of the electrospun nanofibers deposited on the rectangular collector having circular ends with slot widths, 5, 7.5, 10 and $20 \mathrm{~mm}$ respectively. The fiber diameter was found to be decreasing with increasing slot width. Average diameters of fibers along with standard deviation are listed in Table 2. Figure 8a depicts the plot showing the mean fiber diameter (collected using rectangular slotted collectors with circular
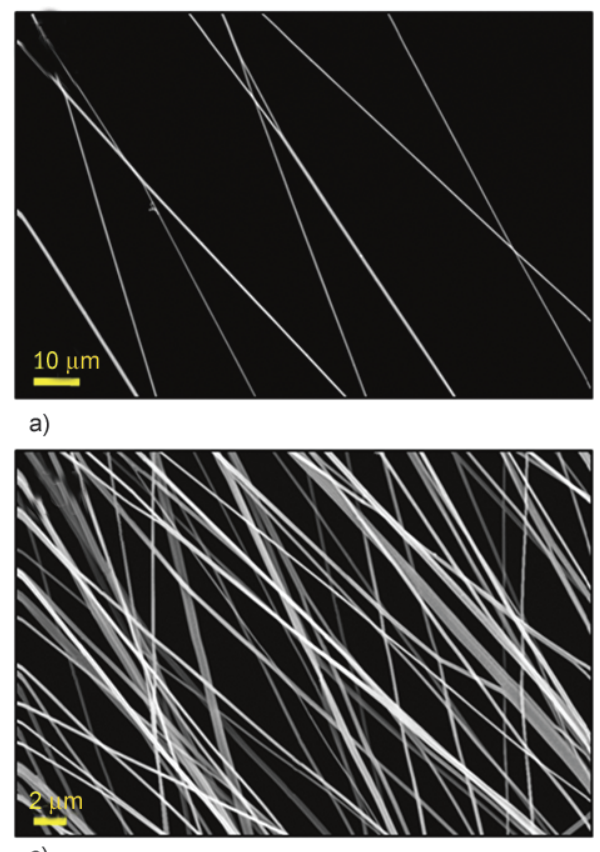

c) ends) vs. slot width. The electrospinning experiments were carried out by fixing the parameters, such as, flow rate, voltage, needle gauge and screen distance and varying the slot width of the collector. The only change introduced was the modification in the electric field; hence the decrease in the fiber diameter must be directly related to the electric field influenced by slot widths. In order to find out the reason for this interesting phenomenon, 2D electrostatic field analysis was carried out for each slot width. The surface plot showing the electric field potential and electric field for rectangular slots with circular ends having $10 \mathrm{~mm}$ slot width is shown in Figure $8 \mathrm{~b}$. The top narrow rectangle represents the electrospinning needle and the two bottom rectangles represent the slotted collector plate in cross section. The top edge of the collector plate was taken as the $X$ coordinate $(-5$ to +5$)$ and the axial direction was taken as the $Y$ coordinate. The maximum value of electric field occurs at the slot edges. The electric field lines bending towards the collector edge is resolved into horizontal $\left(E_{\mathrm{x}}\right)$ and vertical $\left(E_{\mathrm{y}}\right)$ components. From the electrostatic field analysis, it was clear that the $E_{\mathrm{x}}$ is maximum at the edges of the slot. It was found that the $X$ component of the electric field $E_{\mathrm{x}}$, increases with increasing slot width as shown in Figure 8d. The $X$ component of the electrostatic force increases with increase in slot width, as the electrostatic force is proportional to

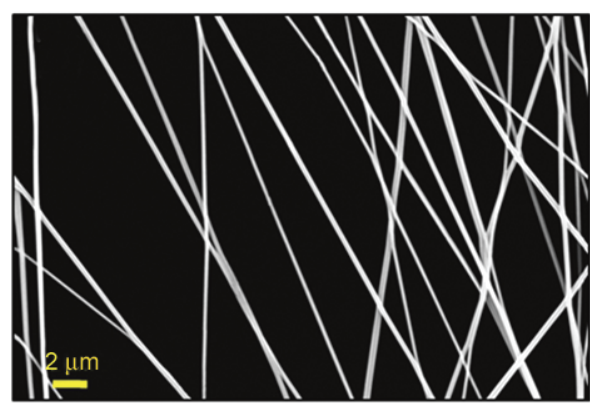

b)

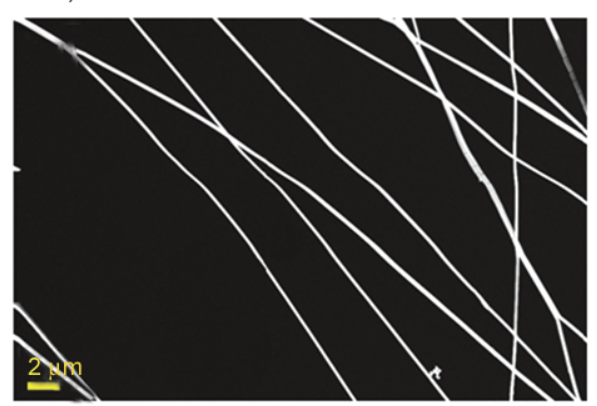

d)

Figure 7. SEM images of aligned nanofibers collected using rectangular slotted collectors with circular ends for the slot width (a) $5 \mathrm{~mm}$, (b) $10 \mathrm{~mm}$, (c) $7.5 \mathrm{~mm}$ and (d) $20 \mathrm{~mm}$ 

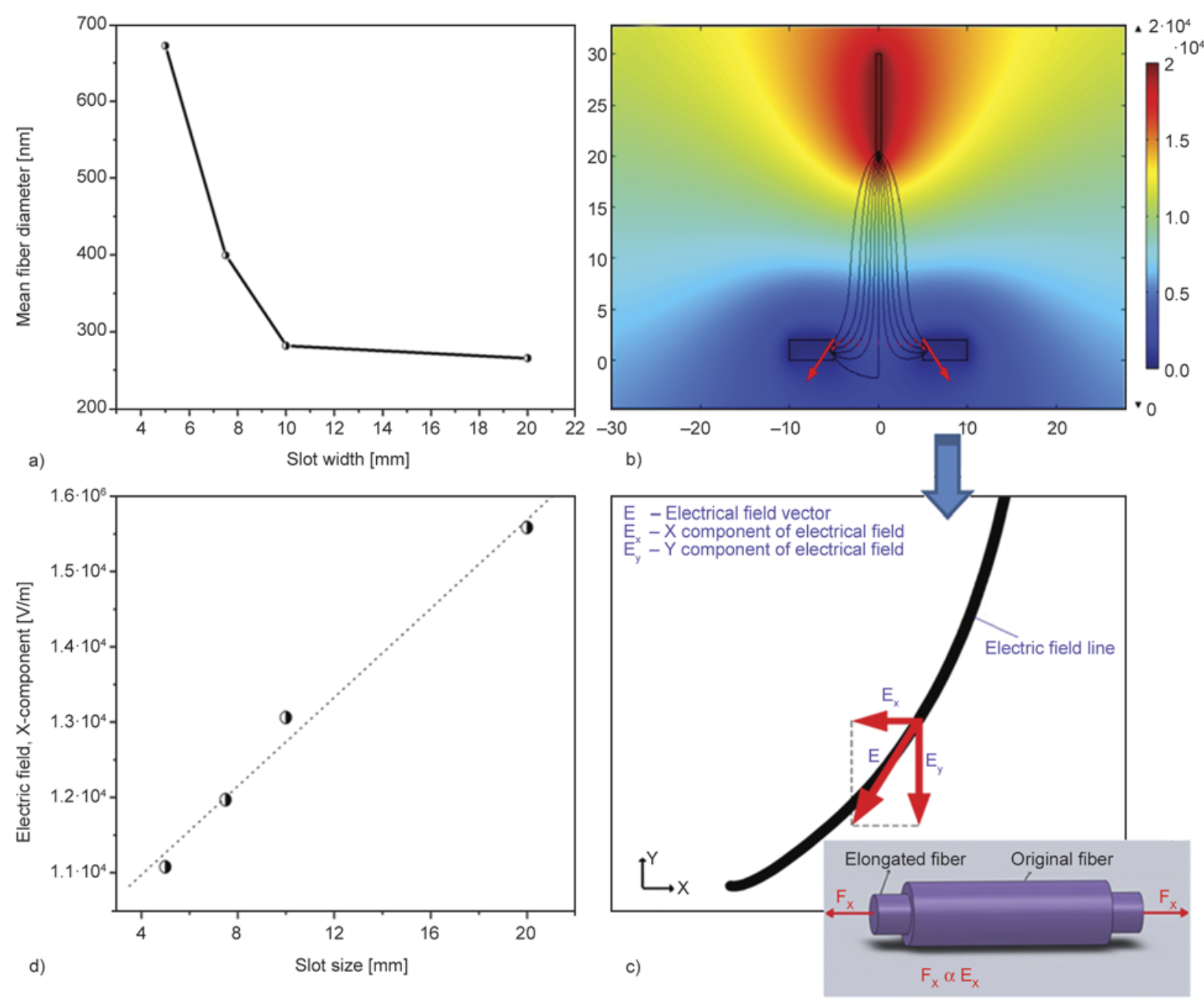

Figure 8. (a) Plot showing the mean fiber diameter (collected using rectangular slotted collectors with circular ends) vs. slot width, (b) surface plot showing electric potential (colour contour) and electric field (arrows and streamlines) for rectangular slotted plates with circular ends having slot width $10 \mathrm{~mm}$, (c) magnified view of a representative electric field line bending towards the collector edge, which has been resolved into horizontal $(X)$ and vertical $(Y)$ components. Inset shows the schematic representation of fiber elongation due to axial elongation and (d) plot showing electric field (maximum values of $X$ component of electric field) as a function of slot widths for rectangular slotted collectors with circular ends

the electric field. Hence, the force along the fiber axis increases with increasing slot size. In Figure 8c, the $X$ component of the electrostatic force acting along the longitudinal axis of the fiber stretches the fiber leading to axial elongation. Whenever there is an axial elongation due to Poisson effect, there will be a lateral contraction leading to a reduction in the fiber diameter. Electrostatic field analysis also revealed that by increasing the slot width the electrostatic force along the fiber axis increases, leading to increase in axial strain which induces further reduction in fiber diameter. This best explains the phenomenon of decreasing fiber diameter with increasing slot widths.
Owing to the excellent electrical conductivity of MWCNT, electrical conductivity was found to be increased upon loading these conductive components into the PVA nanofibers. Figure 9 depicts the current-voltage $(I-V)$ characteristics of the insulating PVA and conducting PVA-MWCNT nanofibers formed using rectangular slot with circular ends. As evident from the figure, the conductivity is significantly increased upon loading $1 \mathrm{wt} \%$ MWCNTs. This may be due to the introduction of MWCNTs into the PVA nanofiber matrix. As evident from TEM analysis, the MWCNTs have aligned parallel to the direction of axis of the nanofiber to form conductive chains during the electrospinning process. 


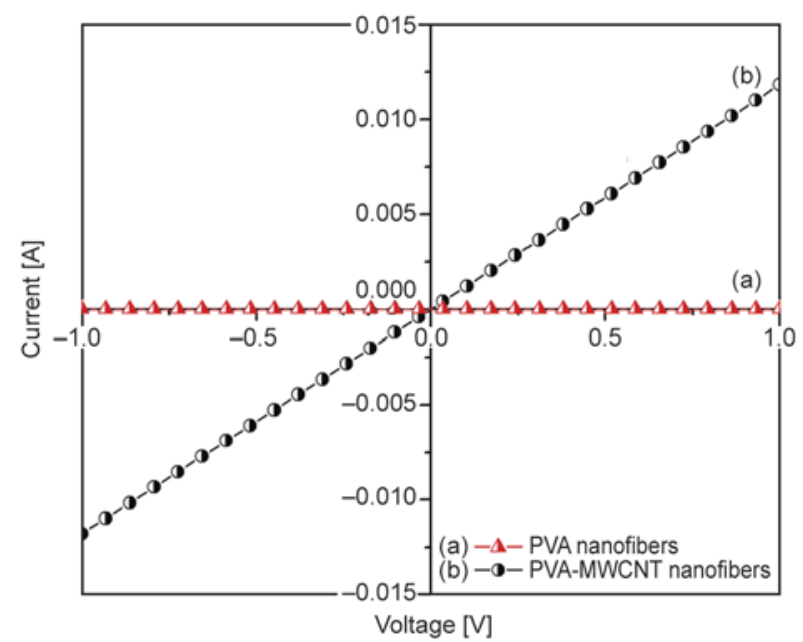

Figure 9. Current-voltage characteristics of (a) PVA nanofibers and (b) PVA-MWCNT nanofibers

The electrical conductivity of PVA-MWCNTs was calculated to be $0.025 \mathrm{~S} / \mathrm{cm}$ from the $I-V$ curves which indicates that the electrospun PVA fibers with aligned conductive components, such as, MWCNTs should be more feasible to conduct electrons when compared with insulating PVA nanofibers and therefore can be ideal candidates for sensor applications. Detailed study on the sensor property analysis of PVA-MWCNT nanofibers is currently ongoing in our laboratory. The investigation shows that the process can be further extended for creating aligned nanofibers onto various transducers having varied geometries and also for biomedical engineering, electronics, and energy storage systems. Apart from that this process can be adopted for the development of a range of materials, such as, polymeric, ceramic and composite based nanofibers. A detailed investigation on the application of this process for microelectrodes is currently investigated in our laboratory for the application of MEMS based sensor devices.

\section{Conclusions}

To summarize, a simple method based on electrostatic field analysis was used for the prediction of alignment of electrospun nanofibers for various collectors having different geometrical shapes. The nature of fiber orientation in each of the collector geometries were predicted and compared with the as-produced electrospun PVA-MWCNT composite nanofibers using electrostatic field analysis. Among all the collector slots, rectangular slotted collectors with circular ends were produced a good fiber alignment over large collecting area. Both the experimental and theoretical studies clearly indicated the intro- duction of an insulating region into a conductive collector and significantly influence the electrostatic forces acting on a charged fiber. PVA-MWCNT nanofibers were found to be conducting in nature owing to the presence of reinforced MWCNTs in PVA matrix. With the aid of electrostatic interactions, electrospun nanofibers could be assembled into controllable structures with varied configurations by varying the collector geometry. One of the most interesting features associated with this approach is that, this technique enables the direct integration of nanofibers with controllable configurations into an electrode system. These nanofibers can be fabricated and aligned simultaneously. This process can significantly simplify the production of nanofiber-based devices for biomedical engineering, electronics, and energy storage systems.

\section{Acknowledgements}

The authors acknowledge for the support and facilities provided by PSG Sons \& Charitable Trust, Coimbatore, INDIA for carrying out the work.

\section{References}

[1] Wang M. C. P., Gates B. D.: Directed assembly of nanowires. Materials Today, 12, 34-43 (2009). DOI: $10.1016 / \mathrm{S} 1369-7021(09) 70158-0$

[2] Vomiero A., Ponzoni A., Comini E., Ferroni M., Faglia G., Sberveglieri G.: Direct integration of metal oxide nanowires into an effective gas sensing device. Nanotechnology, 21, 145502/1-145502/8 (2010). DOI: $10.1088 / 0957-4484 / 21 / 14 / 145502$

[3] Li D., Hu J., Wu R., Lu J. G.: Conductometric chemical sensor based on individual $\mathrm{CuO}$ nanowires. Nanotechnology, 21, 485502/1-485502/6 (2010). DOI: $10.1088 / 0957-4484 / 21 / 48 / 485502$

[4] Wang P., Zhao X., Li B.: ZnO-coated $\mathrm{CuO}$ nanowire arrays: Fabrications, optoelectronic properties, and photovoltaic applications. Optics Express, 19, 11271-11279 (2011). DOI: $10.1364 /$ OE.19.011271

[5] Ramakrishna S, Fujihara K., Teo W-E., Yong T., Ma Z., Ramaseshan R.: Electrospun nanofibers: Solving global issues. Materials Today, 9, 40-50 (2006). DOI: 10.1016/S1369-7021(06)71389-X

[6] Ding B., Wang M., Wang X., Yu J., Sun G.: Electrospun nanomaterials for ultrasensitive sensors. Materials Today, 13, 16-27 (2010). DOI: 10.1016/S1369-7021(10)70200-5

[7] Teo W. E., Ramakrishna S.: A review on electrospinning design and nanofibre assemblies. Nanotechnology, 17, R89-R106 (2006).

DOI: $10.1088 / 0957-4484 / 17 / 14 / R 01$ 
[8] Lukáš D., Sarkar L., Martinová, Vodsed'álková K., Lubasová D., Chaloupek J., Pokorný P., Mikeš P., Chvojka J., Komárek M.: Physical principles of electrospinning (Electrospinning as a nano-scale technology of the twenty-first century). Textile Progress, 41, 59-140 (2009).

DOI: $10.1080 / 00405160902904641$

[9] Bisht G. S., Canton G., Mirsepassi A., Kulinsky L., Oh S., Rankin D-D., Madou M. J.: Controlled continuous patterning of polymeric nanofibers on three-dimensional substrates using low-voltage near-field electrospinning. Nano Letters, 11, 1831-1837 (2011).

DOI: $10.1021 / \mathrm{nl} 2006164$

[10] Yu D-G., Yu J-H., Chen L., Williams G. R., Wang X.: Modified coaxial electrospinning for the preparation of high-quality ketoprofen-loaded cellulose acetate nanofibers. Carbohydrate Polymers, 90, 1016-1023 (2012).

DOI: $10.1016 /$ j.carbpol.2012.06.036

[11] Yu D-G., Liu F., Cui L., Liu Z-P., Wang X., Bligh S. W. A.: Coaxial electrospinning using a concentric Teflon spinneret to prepare biphasic-release nanofibers of helicid. RSC Advances, 3, 17775-17783 (2013). DOI: $10.1039 / C 3 R A 43222 J$

[12] Cavaliere S., Subianto S., Savych I., Jones D. J., Rozière J.: Electrospinning: Designed architectures for energy conversion and storage devices. Energy and Environmental Science, 4, 4761-4785 (2011). DOI: $10.1039 / \mathrm{C} 1 \mathrm{EE} 02201 \mathrm{~F}$

[13] Teo W-E., Ramakrishna S.: Electrospun nanofibers as a platform for multifunctional, hierarchically organized nanocomposite. Composites Science and Technology, 69, 1804-1817 (2009)

DOI: 10.1016/j.compscitech.2009.04.015

[14] Huang Z-M., Zhang Y-Z., Kotaki M., Ramakrishna S.: A review on polymer nanofibers by electrospinning and their applications in nanocomposites. Composites Science and Technology, 63, 2223-2253 (2003). DOI: 10.1016/S0266-3538(03)00178-7

[15] Orlova Y., Magome N., Liu L., Chen Y., Agladze K.: Electrospun nanofibers as a tool for architecture control in engineered cardiac tissue. Biomaterials, 32, 56155624 (2011).

DOI: 10.1016/j.biomaterials.2011.04.042

[16] Carnell L. S., Siochi E. J., Holloway N. M., Stephens R. M., Rhim C., Niklason L. E., Clark R. L.: Aligned mats from electrospun single fibers. Macromolecules, 41, 5345-5349 (2008).

DOI: $10.1021 / \mathrm{ma} 8000143$

[17] Jana S., Cooper A., Ohuchi F., Zhang M.: Uniaxially aligned nanofibrous cylinders by electrospinning. Applied Materials and Interfaces, 4, 4817-4824 (2012). DOI: $10.1021 / \mathrm{am} 301803 \mathrm{~b}$

[18] Khamforoush M., Mahjob M.: Modification of the rotating jet method to generate highly aligned electrospun nanofibers. Materials Letters, 65, 453-455 (2011). DOI: 10.1016/j.matlet.2010.10.060
[19] Theron A., Zussman E., Yarin A. L.: Electrostatic fieldassisted alignment of electrospun nanofibres. Nanotechnology, 12, 384-390 (2001).

DOI: $10.1088 / 0957-4484 / 12 / 3 / 329$

[20] Huang Y., Duan X., Wei Q., Lieber C. M.: Directed assembly of one-dimensional nanostructures into functional networks. Science, 291, 630-633 (2001).

DOI: $10.1126 /$ science.291.5504.630

[21] Katta P., Alessandro M., Ramsier R. D., Chase G. G.: Continuous electrospinning of aligned polymer nanofibers onto a wire drum collector. Nano Letters, 4 , 2215-2218 (2004).

DOI: $10.1021 / \mathrm{n} 10486158$

[22] Chvojka J., Hinestroza J. P., Lukas D.: Production of poly(vinylalcohol) nanoyarns using a special saw-like collector. Fibres and Textiles in Eastern Europe, 21, 28-31 (2013).

[23] Yang L., Yuan W., Zhao J., Ai F., Chen X., Zhang Y.: A novel approach to prepare uniaxially aligned nanofibers and longitudinally aligned seamless tubes through electrospinning. Macromolecular Materials and Engineering, 297, 604-608 (2012). DOI: $10.1002 /$ mame.201100195

[24] Wang Y., Wang G., Chen L., Li H., Yin T., Wang B., Lee J. C-M., Yu Q.: Electrospun nanofiber meshes with tailored architectures and patterns as potential tissueengineering scaffolds. Biofabrication, 015001/1015001/9 (2009). DOI: $10.1088 / 1758-5082 / 1 / 1 / 015001$

[25] Xie J., MacEwan M. R., Ray W. Z., Liu W., Siewe D. Y., Xia Y.: Radially aligned, electrospun nanofibers as dural substitutes for wound closure and tissue regeneration applications. ACS Nano, 4, 5027-5036 (2010). DOI: $10.1021 / \mathrm{nn} 101554 \mathrm{u}$

[26] de Volder M. F. L., Tawfick S. H., Baughman R. H., Hart A. J.: Carbon nanotubes: Present and future commercial applications. Science, 339, 535-539 (2013). DOI: $10.1126 /$ science.1222453

[27] Spitalsky Z., Tasis D., Papagelis K., Galiotis C.: Carbon nanotube-polymer composites: Chemistry, processing, mechanical and electrical properties. Progress in Polymer Science, 35, 357-401 (2010). DOI: $10.1016 / \mathrm{j}$. progpolymsci.2009.09.003

[28] Jeong J. S., Moon J. S., Jeon S. Y., Park J. H., Alegaonkar P. S., Yoo J. B.: Mechanical properties of electrospun PVA/MWNTs composite nanofibers. Thin Solid Films, 515, 5136-5141 (2007).

DOI: $10.1016 / \mathrm{j} . \mathrm{tsf} .2006 .10 .058$

[29] Ge J. J., Hou H., Li Q., Graham M. J., Greiner A., Reneker D. H., Harris F. W., Cheng S. Z. D.: Assembly of well-aligned multiwalled carbon nanotubes in confined polyacrylonitrile environments: Electrospun composite nanofiber sheets. Journal of the American Chemical Society, 126, 15754-15761 (2004).

DOI: $10.1021 / \mathrm{ja} 048648 \mathrm{p}$ 
[30] Dror Y., Salalha W., Khalfin R. L., Cohen Y., Yarin A. L., Zussman E.: Carbon nanotubes embedded in oriented polymer nanofibers by electrospinning. Langmuir, 19, 7012-7020 (2003).

DOI: $10.1021 / 1 \mathrm{a} 034234 \mathrm{i}$

[31] Yeo L. Y., Friend J. R.: Electrospinning carbon nanotube polymer composite nanofibers. Journal of Experimental Nanoscience, 1, 177-209 (2006). DOI: $10.1080 / 17458080600670015$

[32] Huang S., Yee W. A., Tjiu W. C., Liu Y., Kotaki M., Boey Y. C. F., Ma J., Liu T., Lu X.: Electrospinning of polyvinylidene difluoride with carbon nanotubes: Synergistic effects of extensional force and interfacial interaction on crystalline structures. Langmuir, 24, 1362113626 (2008). DOI: $10.1021 / 1 \mathrm{la} 8024183$

[33] Chen D., Liu T., Zhou X., Tjiu W. C., Hou H.: Electrospinning fabrication of high strength and toughness polyimide nanofiber membranes containing multiwalled carbon nanotubes. The Journal of Physical Chemistry B, 113, 9741-9748 (2009). DOI: 10.1021/jp9025128

[34] Zhang Q., Chang Z., Zhu M., Mo X., Chen D.: Electrospun carbon nanotube composite nanofibres with uniaxially aligned arrays. Nanotechnology, 18, 115611/1-115611/7 (2007). DOI: $\underline{10.1088 / 0957-4484 / 18 / 11 / 115611}$
[35] Yee W. A., Nguyen A. C., Lee P. S., Kotaki M., Liu Y., Tan B. T., Mhaisalkar S., Lu X.: Stress-induced structural changes in electrospun polyvinylidene difluoride nanofibers collected using a modified rotating disk. Polymer, 49, 4196-4203 (2008).

DOI: $10.1016 /$ j.polymer.2008.07.032

[36] Yang D., Lu B., Zhao Y., Jiang X.: Fabrication of aligned fibrous arrays by magnetic electrospinning. Advanced Materials, 19, 3702-3706 (2007).

DOI: $10.1002 /$ adma.200700171

[37] Tamura T., Kawakami H.: Aligned electrospun nanofiber composite membranes for fuel cell electrolytes. Nano Letters, 10, 1324-1328 (2010). DOI: $10.1021 / \mathrm{nl} 1007079$

[38] Rosca I. D., Watari F., Uo M., Akasaka T.: Oxidation of multiwalled carbon nanotubes by nitric acid. Carbon, 43, 3124-3131 (2005). DOI: $10.1016 /$ j.carbon.2005.06.019

[39] Li D., Wang Y., Xia Y.: Electrospinning of polymeric and ceramic nanofibers as uniaxially aligned arrays. Nano Letters, 3, 1167-1171 (2003).

DOI: $10.1021 / \mathrm{n} 10344256$ 